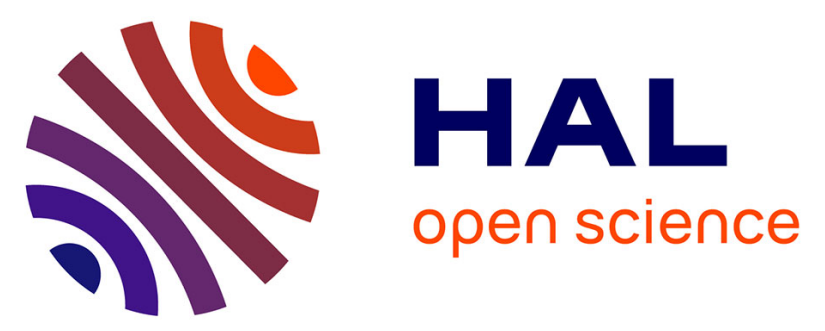

\title{
Levitating spherical particle in a slightly tapered tube at low Reynolds numbers: Application to the low-flow rate rotameters
}

\author{
Stéphane Champmartin, Abdelhak Ambari, R. P. Chhabra
}

\section{To cite this version:}

Stéphane Champmartin, Abdelhak Ambari, R. P. Chhabra. Levitating spherical particle in a slightly tapered tube at low Reynolds numbers: Application to the low-flow rate rotameters. Review of Scientific Instruments, 2012, 83 (12), pp.125103.1-125103.7. 10.1063/1.4769053 . hal-01061933

\section{HAL Id: hal-01061933 \\ https://hal.science/hal-01061933}

Submitted on 8 Sep 2014

HAL is a multi-disciplinary open access archive for the deposit and dissemination of scientific research documents, whether they are published or not. The documents may come from teaching and research institutions in France or abroad, or from public or private research centers.
L'archive ouverte pluridisciplinaire HAL, est destinée au dépôt et à la diffusion de documents scientifiques de niveau recherche, publiés ou non, émanant des établissements d'enseignement et de recherche français ou étrangers, des laboratoires publics ou privés. 


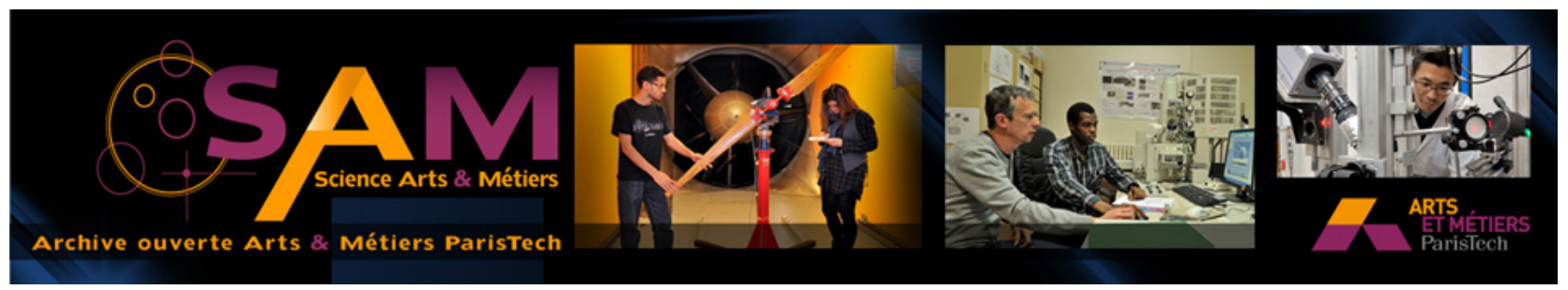

Science Arts \& Métiers (SAM)

is an open access repository that collects the work of Arts et Métiers ParisTech researchers and makes it freely available over the web where possible.

This is an author-deposited version published in: http://sam.ensam.eu

Handle ID: .http://hdl.handle.net/10985/8482

\section{To cite this version :}

Stéphane CHAMPMARTIN, Abdelhak AMBARI, R. P. CHHABRA - Levitating spherical particle in a slightly tapered tube at low Reynolds numbers: Application to the low-flow rate rotameters Review of Scientific Instruments - Vol. 83, n¹2, p.125103.1-125103.7 - 2012 


\title{
Levitating spherical particle in a slightly tapered tube at low Reynolds numbers: Application to the low-flow rate rotameters
}

\author{
S. Champmartin, ${ }^{1, \text { a) }}$ A. Ambari, ${ }^{1}$ and R. P. Chhabra ${ }^{2}$ \\ ${ }^{1}$ Arts et Métiers ParisTech, 2 bd du Ronceray, Angers Cedex 01, 49035, France \\ ${ }^{2}$ Department of Chemical Engineering, Indian Institute of Technology, Kanpur 208016, India
}

\begin{abstract}
In this study, a theoretical framework is developed to predict the equilibrium conditions of a nonneutrally buoyant sphere placed in a vertical conical tube as encountered in liquid rotameters. The analysis presented herein is applicable for a sphere heavier than the surrounding fluid, situated on the axis of a slightly tapered tube. The sphere is subject to the laminar flow conditions with the Reynolds numbers ranging between the Stokes type regimes up to values corresponding to slightly inertial regimes. In this work, we assume that the aperture angle of the tube is small and that the drag force is mainly due to the dissipation located in the gap between the tube and the sphere. Under these conditions, it is possible to consider the tube as locally cylindrical and we can use the results previously obtained for the correction factor of the Stokes force on a sphere subject to a Poiseuille flow in a tube of constant cross-section. We obtain an equation relating the flow rate to the vertical position of the sphere in the tube and the validity of this analysis is demonstrated by applying it to a commercially available rotameter. The present study provides a simple but sound theoretical method to calibrate such flowmeters.

[http://dx.doi.org/10.1063/1.4769053]
\end{abstract}

\section{INTRODUCTION}

Within the broad class of bulk flow metering devices, rotameters constitute an important type of device. The term "rotameter" was derived from the fact that floats originally were produced with slots to give them rotation for the purpose of centering and stabilizing them at the axis of a tube with an upward flow of fluid in it. We restrict ourselves here to the rotameters used for the measurement of low to very low flow rates. This precision instrument is known for its simplicity and ease of operation, requiring little maintenance, and for its versatility and economy. It is popular because it requires no external power, can be positioned near pipe bends, and leads to relatively low pressure losses, but must be mounted vertically with upward flow in the tube. It is particularly suitable for metering carrier gases in chromatographic columns, monitoring and controlling gas flow rates in manufacturing processes, liquid and gas measurement in laboratories, pilot plants, flow and level indicating, etc. It consists of a vertical metering tube and a float. In modern practice, the tubes are formed on a mandrel and annealed to prevent internal stresses so that strong, uniform tubes result. This enables one also to form tubes with better reproducibility and interchangeability or with special shapes, such as non-conical tubes with curved elements designed to spread out the graduations at the lower end of the flow rate range. It is possible to modify the conical form slightly in order to give the exact linear relationship between the flow rate and the float position, which is not possible with a conical tube, as will be seen below. Some additional features can be added, such as the transduction of the flow rate to an electrical output signal (Lui et al. ${ }^{1}$ ), etc. Depending upon

\footnotetext{
a) Author to whom correspondence should be addressed. Electronic mail: stephane.champmartin@ensam.eu.
}

the nature of the process fluid, the float can be made from several materials to avoid corrosion, abrasion, or for the purpose of capacity modification. For low flow rates, the float is generally a small sphere and hence the analysis presented in this work is naturally relevant to this instrument. In this case, the classical float materials are glass, sapphire, stainless steel, tantalum, or carboloy (an alloy of tungsten with cobalt or nickel as a binder). Further information on the variable area flowmeters or rotameters can be found in many practical books such as Goldstein, ${ }^{2}$ Webster $^{3}$, etc.

In addition, it is appropriate to add here that the flow past a sphere mounted on the axis of a tapered cylindrical tube also denotes an idealization of several other industrial applications. Typical examples include the flow of mineral, food, and other suspensions in converging sections of venturimeter, during the emptying of storage and mixing vessels through conical-shaped bottoms, chromatograph-based separations, etc. Hence, it is readily agreed that a satisfactory understanding of the hydrodynamic forces exerted on a sphere in a tapered tube is germane to the understanding and the modeling of the aforementioned applications. However, the major thrust of the present work is to develop a theoretical framework based on numerical and experimental results for the operation and the calibration of a rotameter for measuring low flow rates of incompressible Newtonian fluids. This theoretical framework consists in assuming the tapered tube as locally cylindrical, but with a local radius depending on the vertical position. This assumption is acceptable as long as the angle of the tube remains very small and because the dissipation is located in a small gap between the float and the tube in such confined situations. This work presents a novel analysis to improve the currently used experimental and empirical approaches to the design, standardization, and calibration of this kind of rotameter. 


\section{FORMULATION AND ANALYSIS}

In the absence of the inertial effects in the governing equations, Stokes (1851) obtained for a spherical particle of radius $a$ placed in an unbounded flow with uniform velocity $V$, the classical expression for the drag force acting on the sphere

$$
F_{S}=6 \pi \mu a V .
$$

In this expression, $\mu$ is the dynamic viscosity of the fluid. Strictly speaking, Eq. (1) is applicable when the Reynolds number, $\operatorname{Re}$ (ratio of inertial to viscous forces) based on the sphere radius is small $\left(\operatorname{Re}=\rho_{f} V a / \mu \ll 1\right.$, where $\rho_{f}$ is the mass density of the fluid). If the particle is close to other particles or to a wall, some corrections for the drag force are required to account for the additional hydrodynamic interactions. In the case of a single sphere fixed on the axis of a cylindrical tube of constant radius $b$ and subject to a Poiseuille flow of maximum velocity $V=V_{\max }$, it is possible to re-write Eq. (1) as follows:

$$
F=F_{S} \lambda(k)
$$

with $\lambda(k)$ the correction factor of the Stokes force depending solely on the confinement parameter $k=a / b$ (as shown schematically in Fig. 1 on the left). $F$ is the actual drag force acting on the sphere. Evidently, due to additional energy dissipation at the wall of the tube, $\lambda(k) \geq 1$ and hence, $F \geq F_{S}$. Among the many studies available for the determination of $\lambda(k)$, one can quote those of Ladenburg, ${ }^{4}$ Faxén, ${ }^{5}$ Wakiya, ${ }^{6}$ Happel and co-workers, ${ }^{7,8}$ Haberman and Sayre, ${ }^{9}$ Bohlin, ${ }^{10}$ or Wang and Skalak. ${ }^{11}$ Most of these analytical studies use the reflection method and, are valid at confinements $k<0.6$ and at low Reynolds numbers. These results have been validated experimentally, among others, by Ambari et al. ${ }^{12}$ in the same range of confinements. Bungay et al. ${ }^{13}$ developed a technique based on the singular perturbation method, combined with the asymptotic expansions derived from the

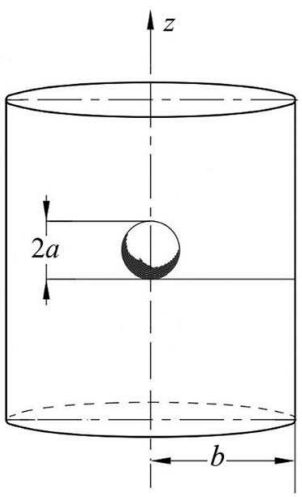

(a)

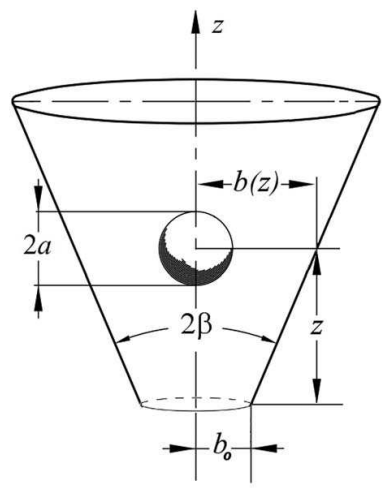

(b)
FIG. 1. Geometrical parameters. analysis of Haberman and Sayre ${ }^{9}$

$$
\begin{aligned}
\lambda(k)= & \frac{3 \pi \sqrt{2}}{16}(1-k)^{-5 / 2}\left[1+\frac{7}{60}(1-k)-\frac{2.227}{50.4}(1-k)^{2}\right] \\
& +\frac{1}{12 \pi}\left[4.018-3.9788 k-1.9215 k^{2}+4.392 k^{3}\right. \\
& \left.+5.006 k^{4}\right] .
\end{aligned}
$$

This formula is valid in the range $0.05<k<0.99$. However, attention is drawn to the fact that the second term in this expression does not vanish for $k \rightarrow 1$ as expected by the asymptotical behavior of $\lambda(k)$, but its value is rather negligible in this limit.

In recent years, numerical values of $\lambda(k)$ have also been reported by Ben Richou et al. ${ }^{14}$ in an extended range of confinement, i.e., $0.05<k<0.98$. Since the relative error between the results of Bungay and Brenner ${ }^{13}$ and those of Ben Richou et $a l .{ }^{14}$ is less than $2 \%$ in this range (as shown by Table III in Ben Richou et al. ${ }^{14}$ ), we can equally use the expression (2) or the interpolated numerical data obtained in our laboratory by Ben Richou et al. ${ }^{14}$ We shall use these results in this work to obtain the condition of levitation of a free sphere suspended in a Poiseuille flow in Stokes type regimes. We consider an upward Poiseuille flow in a vertical cylindrical tube in which we place a non-neutrally buoyant sphere. If the density of the solid particle is greater than that of the fluid, it is possible to find a steady equilibrium position for which the apparent weight of the sphere counterbalances the drag force acting upon the particle, thereby leading to its levitation:

$$
\frac{4}{3} \pi a^{3} \Delta \rho g=6 \pi \mu a V_{\max } \lambda(k)
$$

with $\Delta \rho=\left(\rho_{p}-\rho_{f}\right)>0, \rho_{p}$ is the density of the particle (the same condition is of course applicable if we consider a vertically downward Poiseuille flow with $\Delta \rho<0$ ). Introducing the flow rate of the liquid $Q=\bar{V} \pi b^{2}$ with $\bar{V}=\left(V_{\max } / 2\right)$ the average velocity, this equation takes the following form:

$$
\frac{Q}{Q^{*}}=\frac{\pi b^{2} \bar{V}}{\pi a^{2} V_{S}}=\frac{1}{2 k^{2} \lambda(k)}=f(k) .
$$

In this equation, the characteristic flow rate $Q^{*}$ $=\pi a^{2} V_{S}=2 \pi a^{4} \Delta \rho g / 9 \mu\left(V_{S}=2 a^{2} \Delta \rho g / 9 \mu\right.$ is the settling velocity of sphere in unbounded fluid at low Reynolds number) corresponds to the flow rate of the backflow induced by this settling particle in an unconfined medium. The nondimensional flow rate $f(k)$ depends solely on the confinement parameter $k$. Based on the studies quoted above (Bungay and Brenner ${ }^{13}$ or Ben Richou et al. ${ }^{14}$ ), this functional relationship between $f(k)$ and $k$ is shown in Fig. 2. In a tube of constant section, the equilibrium position of the particle is neutral, i.e., as soon as Eq. (3) is satisfied (neglecting end effects), the sphere will levitate at all values of $z$. In order to obtain a stable equilibrium position, it is necessary to relate the vertical position of the sphere to its local confinement in the tube. One way to achieve this objective is to use a tube of a monotonically varying cross-section. Moreover, to lift the sphere, the initial drag force must overcome its apparent weight. If the cross-section of the tube increases in the flow 


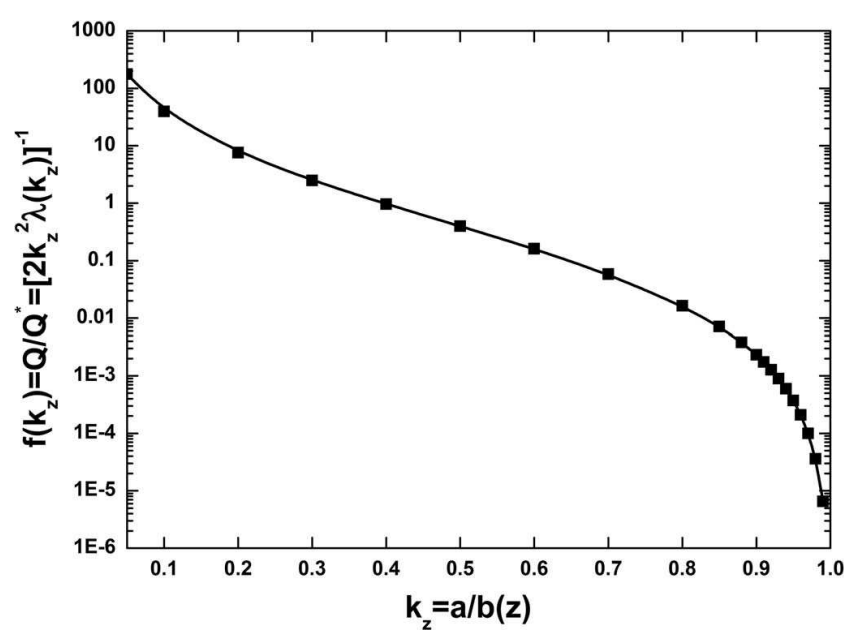

FIG. 2. Non-dimensional flow rate $\mathrm{f}\left(\mathrm{k}_{\mathrm{z}}\right) \mathrm{vs} \mathrm{k}_{\mathrm{z}}=\mathrm{a} / \mathrm{b}(\mathrm{z})$.

direction, the drag force decreases due to the decreasing extent of the confinement effect and a stable equilibrium position can be reached and the levitation achieved. On the contrary, if the cross-section decreases in the flow direction, evidently no such equilibrium position is possible. The simplest shape that fulfills these conditions corresponds to a tapered tube whose radius $b(z)$ increases with the vertical coordinate $z$ (Fig. 1 on the right is exaggerated for clarity). In the limit of small values of the taper angle $\beta$, we can locally consider this system as a sphere confined in a cylindrical tube with its radius given by $b(z)$ (the validity of this hypothesis is studied in Sec. III). Under this assumption, the equilibrium position is still a solution of the Eq. (3) in which the confinement parameter is now a function of the vertical position of the sphere $k=k_{z}=a / b(z)$. Thus we can re-write Eq. (4) as

$$
\frac{Q}{Q^{*}}=\frac{1}{2 k_{z}^{2} \lambda\left(k_{z}\right)}=f\left(k_{z}\right)
$$

For a known functional dependence of the tube radius $b(z)$ on the height $z$ measured from the base of the tube, this expression provides a very simple way to link the flow rate $Q$ in the tube to the position $z$ corresponding to the stable equilibrium position of the sphere. In essence, this is the underlying key principle of the operation of the rotameters at low flow rates or Reynolds numbers (Stokes flow regime). The present work provides a simple method to calibrate such flowmeters as demonstrated in Sec. III.

\section{CALIBRATION AND ANALYSIS}

In order to verify our analysis, we have used a "Type $S$ " low flow rate rotameter obtained from Aalborg Instruments $\&$ Controls ${ }^{\mathrm{TM}}$. The metering tube $\left(\mathrm{N}^{\circ} 112-02-\mathrm{N}\right)$ is a thick walled borosilicate glass tube with a millimeter scale ranging from $z=0 \mathrm{~mm}$ to $z=150 \mathrm{~mm}$. According to the manufacturer, the stated accuracy of the flow rate $Q$ is $\pm 2 \%$ of full scale for this specific type of tube. The inner radius $b(z)$ at a given graduation $z$ and the taper angle $\beta$ of the tube are however unknown (possibly for proprietary reasons). They are not directly accessible because the metering part of the

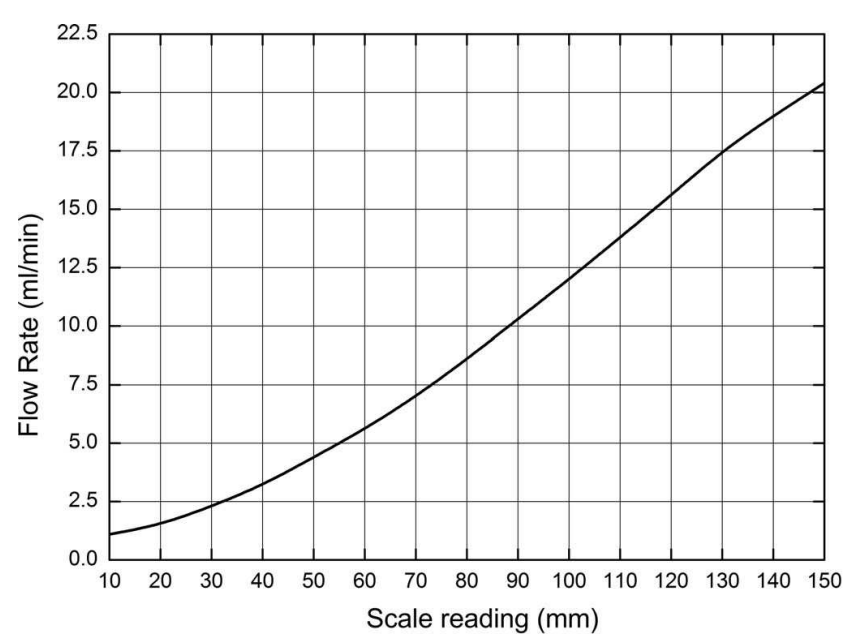

FIG. 3. The 112-02-N tube calibration curve for water and a $1 / 16 /$ radius steel float.

tapered tube is placed between two ends with complex crosssections for the purpose of blocking the float inside the tube and holding it in its rack. However, the manufacturer provides for this specific rotameter the calibration curve shown in Fig. 3 for water at $T=21.1 \pm 0.1{ }^{\circ} \mathrm{C}\left(70^{\circ} \mathrm{F}\right)$ and a stainless steel float of radius $a=1.588 \pm 0.005 \mathrm{~mm}\left(1 / 16^{\prime \prime}\right)$ and density $\rho_{p}=8040 \pm 10 \mathrm{~kg} / \mathrm{m}^{3}$. It is interesting to note that the flow rate is clearly not proportional to the float position, nor does the curve pass through the origin $(0,0)$ point in Fig. 3. First of all, we assume that the tube is an ideal tapered tube such that

$$
b(z)=b_{0}+\tan (\beta) z,
$$

$b_{0}$ being the inner radius at $z=0$ and $\beta$ the angular aperture of the cone. The procedure adopted here is as follows: since our knowledge of the geometrical parameters of the tube is incomplete, we use the calibration curve shown in Fig. 3 to estimate the parameters $b_{0}$ and $\beta$. In a second step, we use these values to calculate a new set of data (flow rate vs position) with a new float material for comparison with the corresponding calibration curve supplied by Aalborg ${ }^{\mathrm{TM}}$.

\section{A. Evaluation of the geometrical parameters of the tube}

We use the relationship given by Eq. (5), i.e.,

$$
\frac{Q}{Q^{*}}=\frac{1}{2 k_{z}^{2} \lambda\left(k_{z}\right)}=f\left(k_{z}\right) \text {. }
$$

In a first step, we evaluate $Q^{*}$ and the accuracy on the various parameters. For water at $T=21.1 \pm 0.1{ }^{\circ} \mathrm{C}$, we have used the density $\rho_{f}=998 \pm 5 \mathrm{~kg} / \mathrm{m}^{3}$ and the viscosity $\mu=0.976 \pm 0.005 \mathrm{mPa} \cdot \mathrm{s}$ (given by the Handbook of Chemistry and Physics ${ }^{15}$ ). The characteristic flow rate $Q^{*}$ depends solely on the float and fluid properties; for the combination of water and a $1 / 16^{\prime \prime}$ radius stainless steel float, we calculate $Q^{*}=(18.83 \pm 0.37) 10^{3} \mathrm{ml} / \mathrm{min}$ taking account of the above uncertainties. Since the accuracy on $Q=Q^{*} f\left(k_{z}\right)=Q^{*} / 2 k_{z}^{2} \lambda\left(k_{z}\right)$ depends on the accuracy of $k_{z}$ (based on the assumption of the locally cylindrical tube) 
TABLE I. Evaluated data of $\mathrm{k}_{\mathrm{z}}$ and $\mathrm{b}(\mathrm{z})$.

\begin{tabular}{lcccr}
\hline \hline$Q(\mathrm{ml} / \mathrm{min})$ & $z(\mathrm{~mm})$ & $k_{z}$ & $b(z)(\mathrm{mm})$ & $\mathrm{Re}$ \\
\hline 1.1 & 10 & 0.976 & 1.627 & 14.5 \\
1.5 & 20 & 0.972 & 1.632 & 19.3 \\
2.3 & 30 & 0.967 & 1.641 & 29.4 \\
3.2 & 40 & 0.963 & 1.649 & 40.5 \\
4.4 & 50 & 0.958 & 1.657 & 55.2 \\
5.6 & 60 & 0.954 & 1.664 & 69.6 \\
7 & 70 & 0.950 & 1.671 & 86.4 \\
8.6 & 80 & 0.946 & 1.679 & 105.2 \\
10.3 & 90 & 0.942 & 1.685 & 124.9 \\
12 & 100 & 0.938 & 1.692 & 144.4 \\
13.8 & 110 & 0.935 & 1.698 & 164.9 \\
15.6 & 120 & 0.932 & 1.703 & 185.2 \\
17.5 & 130 & 0.929 & 1.709 & 206.4 \\
19 & 140 & 0.927 & 1.713 & 223.1 \\
20.4 & 150 & 0.925 & 1.717 & 238.5 \\
\hline \hline
\end{tabular}

and on the one of $\lambda\left(k_{z}\right)$ (based on the numerical results or on an equation such as Eq. (2)), the estimation of the maximum relative uncertainty on the flow rate is $4 \%$ for our analysis (this value is comparable to the $2 \%$ accuracy given by the manufacturer in the calibration data sheet).

The next step consists in using the calibration curve (Fig. 3) to evaluate, for a given flow rate $Q$ (corresponding to a given value of $z$ ), the value of the function $f\left(k_{z}\right)=Q / Q^{*}$. Then the characteristic curve (Fig. 2) or an appropriate expression (see Eq. (7) further) is employed to estimate the local confinement parameter $k_{z}=a / b(z)$. Finally, $b(z)=a / k_{z}$ is deduced. The results are summarized in Table I. It is remarkable that the confinement parameter $k_{z}$ varies very slightly $(\sim 5 \%)$ between the top and the bottom of the tube thereby suggesting only a very small angle of taper and that the confinement is very severe $\left(k_{z} \sim 1\right)$. Hence, only the very last part of Fig. 2 is useful for the present rotameter tube. For such severe confinements, the first part of Eq. (2) is sufficient to accurately calculate $\lambda(k)$ :

$$
\begin{aligned}
\lambda(k) \approx & \frac{3 \pi \sqrt{2}}{16}(1-k)^{-5 / 2} \\
& \times\left[1+\frac{7}{60}(1-k)-\frac{2.227}{50.4}(1-k)^{2}\right] .
\end{aligned}
$$

Therefore, when $k>0.92$ as is the case for the rotameter used here, it is convenient to combine Eqs. (4) and (6) to rewrite the equilibrium condition as follows:

$$
\begin{aligned}
\frac{Q}{Q^{*}} & =f\left(k_{z}\right) \\
& =\frac{8}{3 \pi \sqrt{2}} \frac{\left(1-k_{z}\right)^{5 / 2}}{k_{z}^{2}} \frac{1}{1+\frac{7}{60}\left(1-k_{z}\right)-\frac{2.227}{50.4}\left(1-k_{z}\right)^{2}} .
\end{aligned}
$$

Assuming a perfectly cone-shaped metering tube, the local tube radius $b(z)$ varies linearly with the graduation $z$. In Fig. 4, we have plotted the calculated values of $b(z)$ according to the float position $z$. The data conform satisfactorily to a

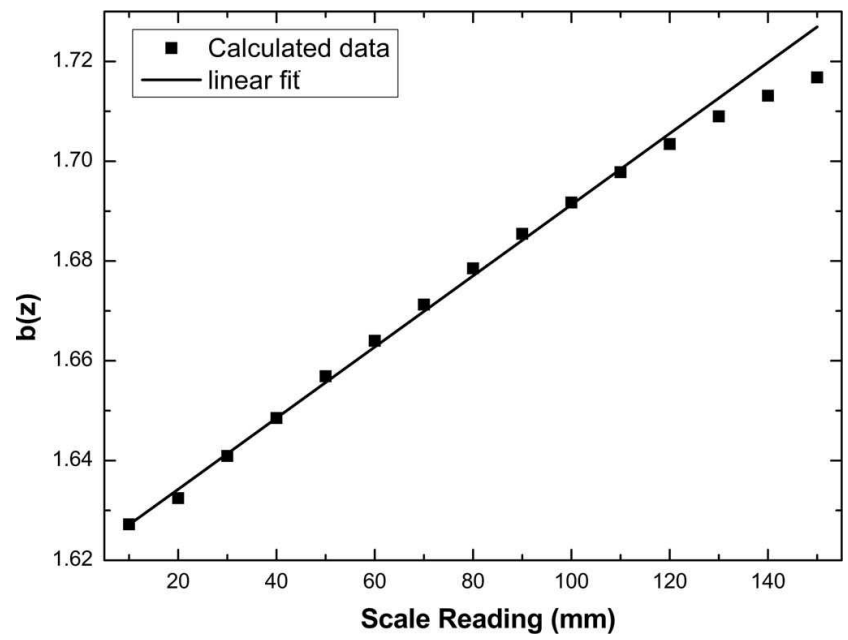

FIG. 4. Local inner radius of the tube vs vertical position.

straight line except for the last three values corresponding to the highest flow rates and the lowest confinements. While the reasons for such a deviation from linear behavior are not immediately obvious, it can probably be ascribed to inertial effects as will be seen later. A linear fit excluding the last three points gives the following values:

$$
b_{0}=1.620 \mathrm{~mm} \text { and } \beta=0.041^{\circ} \text {. }
$$

The very low value of the angle $\beta$ confirms the assumption that the local quasi-constant section tube used in the preceding analysis is justified here. For a vertical displacement of the order of the float radius $\Delta z=a=1.588 \mathrm{~mm}$, the inner radius of the tube varies by an amount given by $\Delta b=a$ tan $\beta=11.3 \mu \mathrm{m}$, which is very small compared to the tube radius. For the lowest value of the radius $b_{0}=1.620 \mathrm{~mm}$, this corresponds to the maximum relative variation of $\Delta b / b_{0}$ $\approx 0.7 \%$ in the tube radius. However, we can also verify that the relative variation of the drag force on the spherical particle for a vertical distance of $\Delta z=a$ is compatible with the value of $\beta$ computed above. Indeed it is possible to calculate:

$$
\frac{\Delta F}{F}=\frac{\tan \beta}{\lambda\left(k_{z}\right)}\left(\frac{d \lambda}{d k_{z}}\right)\left(\frac{a \Delta z}{b^{2}}\right)=\frac{\tan \beta}{\lambda\left(k_{z}\right)}\left(\frac{d \lambda}{d k_{z}}\right) k_{z}^{2} .
$$

Since $0.925<k_{z}<0.976$ (see Table I), we can calculate for example the angle $\beta$, which would give a $1 \%$ relative variation of $F$. We find that $\beta<0.02^{\circ}$. Besides, we must emphasize that for such strong confinements, most of the drag force is due to the dissipation located in the small gap between the particle and the tube. Moreover, the higher the confinement, the smaller this region (see for instance Fig. 11 in Ben Richou et $\left.a l .{ }^{14}\right)$. This implies that the vertical distance $\Delta z$ to consider in the above calculation is, by far, less than the sphere radius $a$ but only by a small fraction $\varepsilon=\Delta z / \Delta a$ of it. Taking the reasonable average value $\varepsilon=10 \%$, we obtain $\beta<0.2^{\circ}$. This is further evidence that the tapered tube can be considered as locally cylindrical for this application. 
TABLE II. Comparison between the calculated values and the calibration data for a glass float in water.

\begin{tabular}{lccc}
\hline \hline$z(\mathrm{~mm})$ & $\begin{array}{c}Q(\mathrm{ml} / \mathrm{min}) \\
(\text { calculated })\end{array}$ & $\begin{array}{c}Q(\mathrm{ml} / \mathrm{min}) \\
(\text { calibration })\end{array}$ & $\begin{array}{c}\text { Relative discrepancy } \\
(\%)\end{array}$ \\
\hline 10 & 0.24 & 0.23 & 3.5 \\
20 & 0.36 & 0.33 & 8.2 \\
30 & 0.51 & 0.49 & 4.2 \\
40 & 0.70 & 0.69 & 0.8 \\
50 & 0.91 & 0.95 & 3.9 \\
60 & 1.17 & 1.24 & 6.0 \\
70 & 1.46 & 1.55 & 5.9 \\
80 & 1.80 & 1.88 & 4.7 \\
90 & 2.17 & 2.31 & 6.5 \\
100 & 2.58 & 2.74 & 6.0 \\
110 & 3.04 & 3.20 & 5.1 \\
120 & 3.55 & 3.71 & 4.6 \\
130 & 4.10 & 4.24 & 3.5 \\
140 & 4.69 & 4.70 & 0.1 \\
150 & 5.34 & 5.50 & 3.0 \\
\hline \hline
\end{tabular}

\section{B. Comparison with another calibration curve}

In order to check the usefulness of these results, we now reconstruct the characteristic curve of the flow rate versus the float position from the estimated values of $b_{0}$ and $\beta$ with a float of the same size, but made of glass $\left(\rho_{p}=2530\right.$ $\pm 5 \mathrm{~kg} / \mathrm{m}^{3}$ ) instead of steel. Water is still the working fluid here. This corresponds to a new value of the characteristic flow rate $Q^{*}=4096 \pm 81 \mathrm{ml} / \mathrm{min}$. The procedure is the same as that employed in Sec. III A but in the reverse order: starting from a given position $z$, we compute the value of $b(z)$ and thus of $k_{z}=a / b(z)$. The function $f\left(k_{z}\right)$, evaluated using an appropriate function (Eq. (7)) or curve (Fig. 2), is computed and finally, the flow rate $Q=Q^{*} f\left(k_{z}\right)$ is calculated. In Table II and Fig. 5 below, these calculated values are compared with the calibration data given by Aalborg ${ }^{\mathrm{TM}}$. We obtain a good agreement between both sets of values with an

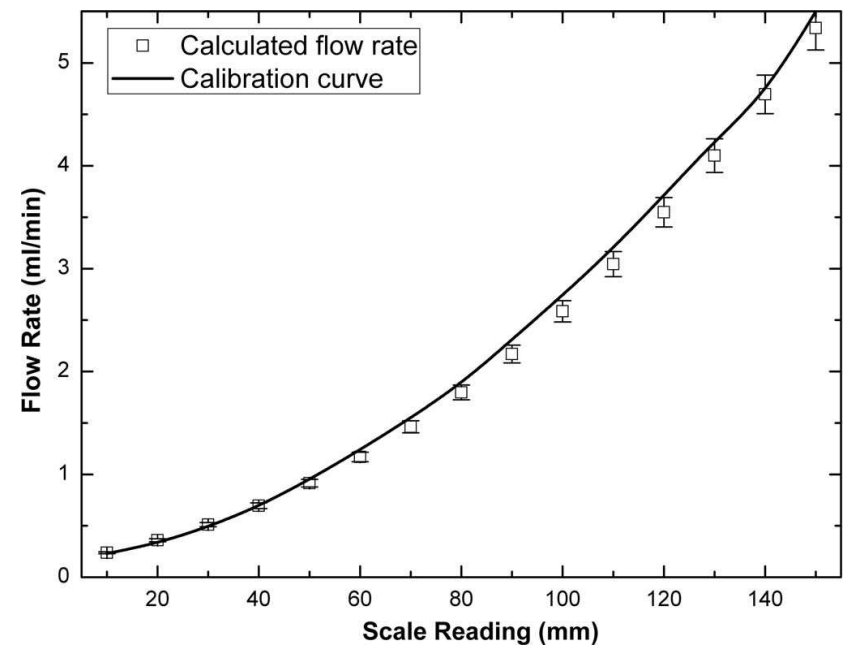

FIG. 5. Comparison between the calculated values and the calibration data for a glass float in water. average relative discrepancy for the flow rate below $5 \%$ with no discernable trends with respect to $z$.

Each value of the calculated flow rate is given with its error bar based on the relative uncertainty $\Delta Q / Q \approx 4 \%$ evaluated with the same approach as at the beginning of Sec. III A.

\section{Influence of the Reynolds number}

In the preceding analysis, we have assumed negligible inertial effects. Nevertheless, it is well known that the Stokes force $F_{S}=6 \pi \mu a \mathrm{~V}$ begins to drift from experimental results as soon as the value of the Reynolds number exceeds $\operatorname{Re}>0.1$ in an unbounded flow. For a confined flow however, it is known that the onset of the influence of inertial effects is somewhat delayed. This problem was thoroughly studied both numerically in our laboratory (see for instance the work of Despeyroux ${ }^{16}$ ), and experimentally by Coutanceau ${ }^{17}$ (their studies clearly show that the onset of the recirculation zone behind the sphere is delayed and its size is reduced as the confinement increases). In order to ascertain the role of inertial effects in the present situation, we have nevertheless done a computational study with the code FLUENT ${ }^{\mathrm{TM}}$. In Fig. 6, the drag force normalized by $F_{S} \lambda(k)$ is plotted as a function of the Reynolds number for confinement parameters in the range 0.4 $<k<0.9$. At low Reynolds numbers, these plots are characterized by plateaus corresponding to the Stokes type regimes for which inertia is negligible followed by a complex evolution depending on the values of the Reynolds number and $k$. Since the stated precision of the calibration curve is $2 \%$ of the full scale, we have arbitrarily taken a criterion ( $5 \%$ increase of the normalized drag force) to estimate the Reynolds numbers corresponding to the "onset" of inertia. The resulting values of the Reynolds number are summarized in Table III. As expected, the larger the confinement, the higher the Reynolds number corresponding to the onset of inertial effects.

The metering tube $\left(\mathrm{N}^{\circ} 112-02-\mathrm{N}\right)$ of Aalborg with the float of radius $a=1 / 16^{\prime \prime}=1.588 \mathrm{~mm}$ gives confinement parameters greater than $k \sim 0.92$ and thus the inertial effects are expected to be negligible for the Reynolds number values even beyond $\operatorname{Re}=233$. For each flow rate $Q$ in Table I, we

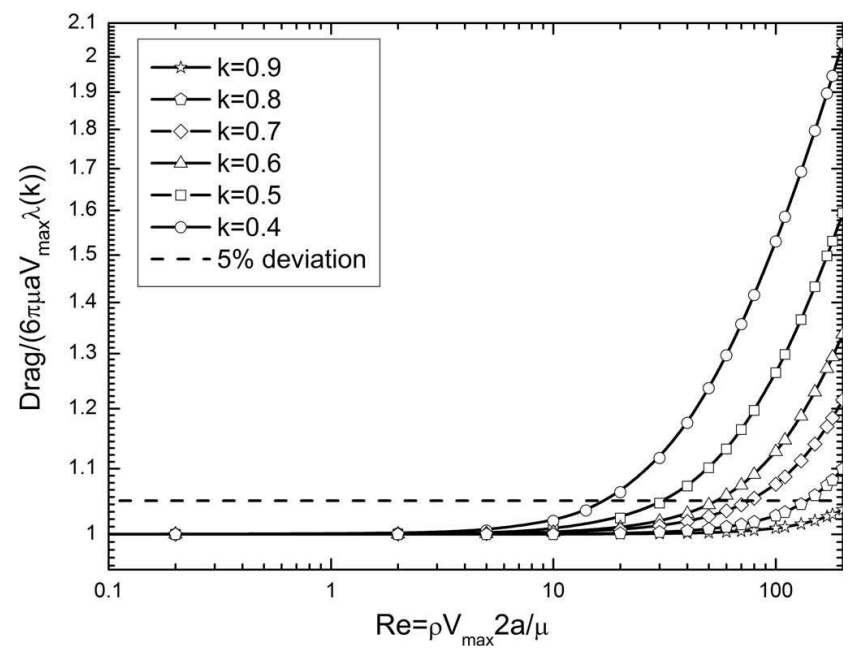

FIG. 6. Influence of inertia on the drag force. 
TABLE III. Estimation of the Reynolds number for the onset of inertia.

\begin{tabular}{cc}
\hline \hline$k$ & $\mathrm{Re}=V_{\max } 2 a / v$ \\
\hline 0.4 & 17 \\
0.5 & 31 \\
0.6 & 54 \\
0.7 & 76 \\
0.8 & 138 \\
0.9 & 233 \\
\hline \hline
\end{tabular}

have included the local Reynolds number corresponding to the value of $b(z)$ at which the float stabilizes as

$$
\operatorname{Re}=\frac{\rho V_{\max } 2 a}{\mu}=\frac{4 \rho Q k_{z}}{\pi b(z) \mu} .
$$

Evidently, the last two or three values in Table I are very close or greater than the critical value of $\mathrm{Re}=233$ and thus it is likely that it is no longer possible to neglect the inertial effects under these conditions. This may also explain, at least in part, why these points do not conform to a linear law in Fig. 4.

\section{Inverse problem}

Until now, we have assumed that the metering tube was tapered with a radius varying linearly with the vertical position. As shown in Figs. 3 and 5, this corresponds to a flow rate $Q$ that is not proportional to the float equilibrium position $z$. We now attempt to find the shape of the metering tube, which would result in a linear relationship between the flow rate and the float position. Let us take the case of a stainless steel float in water. Considering the numerical data in Table I, we see that the flow rate varies between $Q=1.1 \mathrm{ml} / \mathrm{min}$ at $z=10 \mathrm{~mm}$ and $Q=20.4 \mathrm{ml} / \mathrm{min}$ at $z=150 \mathrm{~mm}$. For comparison purposes, we can evaluate the average rate of change of the flow rate with $z$, denoted by $\alpha=\Delta Q / \Delta z$, the mean value of which is evaluated as

$$
\alpha=\frac{\Delta Q}{\Delta z}=\frac{19.3}{140} \approx 0.138 \mathrm{ml} / \mathrm{min} \cdot \mathrm{mm} .
$$

Hence, the flow rate obeys the equation

$$
Q(z)=1.1+\alpha(z-10) \mathrm{in} \mathrm{ml} / \mathrm{min} \text { with } z \text { in } \mathrm{mm} .
$$

From the value of $Q(z)$ at a given position $z$, we can evaluate $k_{z}=a / b(z)$ and then $b(z)$ and these results are presented in Table IV below. The calculated values of the Reynolds number show also that the last three points corresponding to the higher flow rates are likely to be less reliable because of the possible influence of inertia.

The hypothetical profile of the tube is shown in Fig. 7 and compared to the uniformly tapered-shaped tube. The crossing of the curves for the highest positions is due to the linear regression calculated above that overestimates the flow rate as can be seen in Fig. 4. In order to have a linear relationship between the flow rate and the float position, it is therefore necessary to make a concave tube whose radius varies more steeply at the bottom than at the top of the tube. This approach could of course be extended to any float, any fluid, or any relation-
TABLE IV. Evolution of $\mathrm{b}(\mathrm{z})$ giving $\mathrm{Q}$ proportional to $\mathrm{z}$.

\begin{tabular}{lcccr}
\hline \hline$Q(\mathrm{ml} / \mathrm{min})$ & $z(\mathrm{~mm})$ & $k_{z}$ & $b(z)(\mathrm{mm})$ & $\mathrm{Re}$ \\
\hline 1.1 & 10 & 0.976 & 1.627 & 14.4 \\
2.5 & 20 & 0.966 & 1.643 & 31.6 \\
3.9 & 30 & 0.960 & 1.653 & 48.6 \\
5.2 & 40 & 0.955 & 1.662 & 65.3 \\
6.6 & 50 & 0.951 & 1.669 & 81.8 \\
8.0 & 60 & 0.947 & 1.676 & 98.0 \\
9.4 & 70 & 0.944 & 1.682 & 114.1 \\
10.8 & 80 & 0.941 & 1.687 & 130.1 \\
12.1 & 90 & 0.938 & 1.692 & 145.9 \\
13.5 & 100 & 0.936 & 1.697 & 161.7 \\
14.9 & 110 & 0.933 & 1.701 & 177.2 \\
16.3 & 120 & 0.931 & 1.705 & 192.6 \\
17.6 & 130 & 0.929 & 1.709 & 208.1 \\
19.0 & 140 & 0.927 & 1.713 & 223.3 \\
20.4 & 150 & 0.925 & 1.717 & 238.5 \\
\hline \hline
\end{tabular}

ship between the flow rate and the float position provided that the Stokes regime is respected.

\section{E. Discussion about the axial position of the particle}

Undoubtedly, the foregoing analysis hinges on the assumption that the spherical float is always at the axis of the tube and therefore it is appropriate to make some comments on this aspect as a small degree of eccentricity can influence the value of $\lambda(k)$. An extensive experimental and analytical body of knowledge exists on the migration of an isolated particle and in a concentrated suspension when subject to a velocity distribution in the tube (see for instance Segré and Silberberg, ${ }^{18}$ Matas et al. ${ }^{19}$ or Eichhorn and Small $\left.{ }^{20}\right)$. Suffice to say here that such a migration of particles across the streamlines is caused by inertial effects, e.g., see the studies of Brenner et al., ${ }^{21}$ Bretherton, ${ }^{22}$ etc. On the other hand, Saffman ${ }^{23,24}$ showed theoretically that a sphere in a shear flow field experiences a lateral force whose direction depends upon the relative velocity between the sphere and the fluid. When the particle velocity is faster than the undisturbed local fluid velocity, the resulting lateral force tends to push the sphere

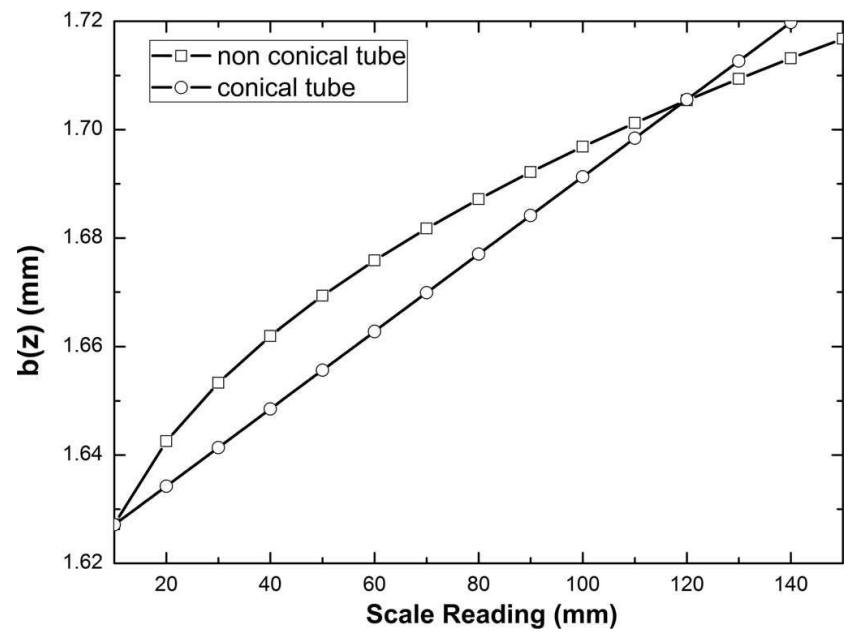

FIG. 7. Radius evolution for a conical and a non-conical tube. 
away from the axis of the tube towards the wall and in the opposite case the sphere migrates towards the axis of the tube. Since in the present analysis $\rho_{p}>\rho_{f}$, the sphere velocity is smaller than the undisturbed velocity and the lateral force on the sphere acts to move it towards the axis of the tube. Thus, the sphere is likely to stay on the axis of the tube over the range of conditions of interest here. However, let us recall that for very high flow rate rotameters, inertial instabilities can lead to radial fluctuations in the sphere position. This effect is usually reduced by the use of guide ribs to prevent any radial displacement.

\section{CONCLUSION}

In this study, we have proposed a new theoretical approach to analyze the stable equilibrium position of a nonneutrally buoyant spherical particle placed on the axis of a tapered tube. We have used the results concerning the correction factor of the Stokes force on a sphere placed on the axis of a cylindrical tube in Poiseuille flow. Assuming that the angle of tapering is small, together with the fact that the dissipation is confined in the very small clearance between the sphere and the tube, we show the relevance of this approach and its usefulness for the practical problem of the variable area flowmeters such as the rotameters at low flow rates in the limit of negligible inertia. However, this restriction does not imply that the particle Reynolds numbers are small, because inertia is drastically reduced in the case of large confinements. Finally, this analysis provides an easy way to calculate the profile of a tube that would give a linear dependence of the flow rate with regards to the vertical position of the sphere or any other dependence law and this may lead to a new and/or improved design of rotameters.

\section{ACKNOWLEDGMENTS}

During the course of this work, Professor R. P. Chhabra was an invited professor at ENSAM, Angers. We would like to thank the administration of ENSAM for providing us with this opportunity, which made this collaboration possible.

${ }^{1}$ C. Y. Liu, K. L. Ng, and A. N. Poo, Rev. Sci. Instrum. 61, 887 (1990).

${ }^{2}$ R. J. Goldstein, Fluid Mechanics Measurements, 2nd ed. (CRC, 1996), p. 314.

${ }^{3}$ J. G. Webster, The Measurement, Instrumentation, and Sensors Handbook, 1st ed. (CRC, 1998), pp. 28-13.

${ }^{4}$ R. Ladenburg, Ann. Phys. 23, 447 (1907)

${ }^{5}$ H. Faxén, Ark. Mat., Astron. Fys. 17, 1 (1922).

${ }^{6}$ S. Wakiya, J. Phys. Soc. Jpn. 8, 254 (1953).

${ }^{7}$ J. Happel and B. J. Byrne, Ind. Eng. Chem. 46, 1181 (1954).

${ }^{8} \mathrm{~J}$. Happel and H. Brenner, Low Reynolds Number Hydrodynamics (Prentice-Hall, Englewood Cliffs, 1965).

${ }^{9}$ W. L. Haberman and R. M. Sayre, David Taylor Model Basin Report No. 1143 (Washington, DC, 1958)

${ }^{10}$ T. Bohlin, Trans. R. Inst. Technol., Stockholm 155, 1 (1960).

${ }^{11}$ H. Wang and R. Skalak, J. Fluid Mech. 38, 75 (1969).

${ }^{12}$ A. Ambari, B. Gauthier-Manuel, and E. Guyon, Phys. Fluids 28(5), 1559 (1985).

${ }^{13}$ P. M. Bungay and H. Brenner, Int. J. Multiphase Flow 1, 25 (1973).

${ }^{14}$ A. Ben Richou, A. Ambari, and J. K. Naciri, Eur. Phys. Appl. Phys. 24, 153 (2003).

${ }^{15}$ Handbook of Chemistry and Physics, 65th ed. (CRC, Boca Raton, Florida, 1984).

${ }^{16}$ A. Despeyroux, Ph.D. dissertation, ENSAM, Angers, 2011.

${ }^{17}$ M. Coutanceau, Ph.D dissertation, University of Poitiers, 1971

${ }^{18}$ G. Segré and A. Silberberg, J. Fluid Mech. 14, 136 (1962).

${ }^{19}$ J. P. Matas, J. J. Morris, and E. Guazzelli, Oil Gas Sci. Technol. 59, 59 (2004).

${ }^{20}$ R. Eichhorn and S. Small, J. Fluid Mech. 20, 513 (1964).

${ }^{21}$ H. Brenner and J. Happel, J. Fluid Mech. 4, 195 (1958).

${ }^{22}$ F. P. Bretherton, J. Fluid Mech. 14, 284 (1962).

${ }^{23}$ P. G. Saffman, J. Fluid Mech. 22, 385 (1965).

${ }^{24}$ P. G. Saffman, J. Fluid Mech. 31, 624 (1968). 